\title{
Approximate and Exact Solutions to Fractional Order Cauchy Reaction-Diffusion Equations by New Combine Techniques
}

\author{
Adnan Khan $\mathbb{D}^{1},{ }^{1}$ Muhammad Imran Liaqat, ${ }^{1}$ Muhammad Younis, ${ }^{2}$ and Ashraful Alam $\mathbb{D i D}^{3}$ \\ ${ }^{1}$ National College of Business Administration \& Economics, Lahore, Pakistan \\ ${ }^{2}$ PUCIT, University of the Punjab, Lahore 54000, Pakistan \\ ${ }^{3}$ Department of Mathematics, Jahangirnagar University, Savar, Dhaka, Bangladesh \\ Correspondence should be addressed to Ashraful Alam; ashraf_math20@juniv.edu
}

Received 9 October 2021; Revised 22 November 2021; Accepted 26 November 2021; Published 16 December 2021

Academic Editor: Fairouz Tchier

Copyright (@) 2021 Adnan Khan et al. This is an open access article distributed under the Creative Commons Attribution License, which permits unrestricted use, distribution, and reproduction in any medium, provided the original work is properly cited.

In this paper, we present a simple and efficient novel semianalytic method to acquire approximate and exact solutions for the fractional order Cauchy reaction-diffusion equations (CRDEs). The fractional order derivative operator is measured in the Caputo sense. This novel method is based on the combinations of Elzaki transform method (ETM) and residual power series method (RPSM). The proposed method is called Elzaki residual power series method (ERPSM). The proposed method is based on the new form of fractional Taylor's series, which constructs solution in the form of a convergent series. As in the RPSM, during establishing the coefficients for a series, it is required to compute the fractional derivatives every time. While ERPSM only requires the concept of the limit at zero in establishing the coefficients for the series, consequently scarce calculations give us the coefficients. The recommended method resolves nonlinear problems deprived of utilizing Adomian polynomials or He's polynomials which is the advantage of this method over Adomain decomposition method (ADM) and homotopy-perturbation method (HTM). To study the effectiveness and reliability of ERPSM for partial differential equations (PDEs), absolute errors of three problems are inspected. In addition, numerical and graphical consequences are also recognized at diverse values of fractional order derivatives. Outcomes demonstrate that our novel method is simple, precise, applicable, and effectual.

\section{Introduction}

Differential equations (DEs) can be resolved by a diversity of procedures, analytical and numerical. However, there are numerous analytic methods for verdict on the results of DEs; there occur quite a numeral of DEs that cannot be explained analytically. This means that the result cannot be articulated as a summation of a fixed numeral of basic functions.

Numerous DEs arising in applications are so thorny that it is occasionally unreasonable to have result formulations or as a minimum if a result formula is existing, it possibly will comprise integrals that can be premeditated only by means of an algebraic quadrature formulation. In moreover instance, numerical procedures offer an influential substitute means for resolving the DEs under the prearranged preliminary condition.
Earlier, numerous procedures have been offered to resolve fractional order DEs comprising the Bernstein wavelets method [1], Shehu variational iteration method [2], Chebyshev spectral collocation approach [3], Taylor wavelet technique [4], operational matrix approach [5], fractional natural decomposition method [6]. homotopy analysis approach [7], Aboodh decomposition approach [8], Sumudu decomposition method [9], Elzaki decomposition technique [10], residual power series method [11], and generalized pseudospectral method [12]. Numerical method is based on the generalized fractional order of the Chebyshev orthogonal functions (GFCFs) and the collocation method [13].

In this research, an easy and effective novel semianalytical method is initiated. The unexploited method is called ERPSM that is the merger of ETM and RPSM. The process of this efficacious method relies on transforming $\mathrm{DE}$ 
into the Elzaki space and creating a series explanation and subsequently acquiring the consequence of the actual DE by utilizing the inverse ETM.

Reaction-diffusion equation is a mathematical model, characterized by the parabolic PDEs. It is exemplifying in what way chemicals might work to each other, whereas they diffuse by a medium instantaneously. Alan Tuning recognized it in 1952 [14]. Reaction-diffusion is measured immensely by experts in biology, chemistry, physics, and computer science [15].

By a reaction-diffusion, we mean an equation of the following form:

$$
\frac{\partial \Phi}{\partial \Upsilon}=\Delta \Phi+\Omega(\Phi, \Delta \Phi, \chi, \Upsilon)
$$

where $\Phi$ is the diffusion term and $\Omega(\Phi, \Delta \Phi, \chi, \Upsilon)$ is the reaction term.

In this paper, we deliberate the one-dimensional timefractional CRDEs. The time-fractional CRDEs can be utilized to explicate several categories of linear and nonlinear systems in physics, chemistry, ecology, biology, and engineering [16-18].

The general form of the fractional order CRDE is as follows [19]:

$$
\begin{array}{r}
\frac{\partial^{\oplus} \Phi(\chi, \Upsilon)}{\partial \Upsilon^{\Phi}}=\lambda \frac{\partial^{2} \Phi(\chi, \Upsilon)}{\partial \chi^{2}}+z(\chi, \Upsilon) \Phi(\chi, \Upsilon) \\
\chi \geq 0, \Upsilon \geq 0,0<\bowtie \leq 1 .
\end{array}
$$

With the initial condition,

$$
\Phi(\chi, 0)=g(\chi) .
$$

Fractional derivative is considered in the Caputo sense. The term $\lambda\left(\partial^{2} \Phi(\chi, \Upsilon) / \partial \chi^{2}\right)$ represents diffusion and $z(\chi, \Upsilon) \Phi(\chi, \Upsilon)$ represents the reaction, where $z(\chi, \Upsilon)$ is the reaction parameter, $\Phi(\chi, \Upsilon)$ is the concentration, and $\lambda$ is the diffusion coefficient constant.

Verdict on the results of fractional order CRDEs is a fascinating zone for the researchers. Chowdhury and Hashim applied homotopy-perturbation method (HPM) to acquire estimated analytical explanations for the CRDEs [20]. Ali et al. established estimated results of CRDEs by optimal homotopy asymptotic method (OHAM) [21]. Wang and Liu used a novel evaluating procedure for nonlinear time-fractional CRDE [22]. Kumar et al. applied homotopy analysis transform method (HATM) for cracking CRDEs [23]. Hosseini et al. recognized comparative explanation of CRDEs by Mittag-Leffler law [24]. Lima et al. considered problems of CRDEs by means of finite element approach [25].

Elzaki transform was presented by Elzaki in 2011 [26]. It is a very useful method to resolve the entire natures of DEs.

Elzaki transform was defined for functions of exponential order. We consider functions in the set $\xi$ defined as

$$
\xi=\left\{\Phi(\Upsilon)\left|\exists M, \Theta_{1}, \Theta_{2}>0,\right| \Phi(\Upsilon)<M e^{\left(|Y| \mid \Theta_{j}\right)} \text { if } \Upsilon \in(-1)^{j} X[0, \infty)\right\} .
$$

Elzaki transform is defined as

$$
E[\Phi(\Upsilon)]=v \int_{0}^{\infty} \Phi(\Upsilon) e^{-(\Upsilon / v)} \mathrm{d} \Upsilon, \quad \Theta_{1} \leq v \leq \Theta_{2},
$$

where $E$ symbolizes Elzaki transform operator.

The framework of this study is as follows. In the next section, a new form of fractional Taylor's series is introduced that will be used in our work in the next sections and further explained and the conditions for convergence of the new form of Taylor's formula were determined. Moreover, we presented some new results. Next, we build Elzaki residual power series solutions for CRDEs. Further, few problems are solved to illustrate the capability, the potentiality, and the simplicity of the proposed method. Eventually, our results are compiled in the conclusion.

\section{Some New Results}

In this section, we familiarize a novel formula of fractional Taylor's series and elucidate and govern the circumstances for the convergence of the novel formula of fractional Taylor's series and present some expedient outcomes which are pillars for the new effectual method.
Lemma 1 (a new formula of fractional Taylor's series in Elzaki transform). Suppose that $\Phi(\Upsilon)$ is a piecewise continuous and exponential order; the Elzaki transform of $\Phi(\Upsilon) E[\Phi(\Upsilon)]=\Psi(v)$ has fractional Taylor's series representation as

$$
\Psi(v)=\sum_{n=0}^{\infty} \aleph_{n} v^{n \omega+2},
$$

where $\aleph_{n}$ represents nth coefficient of the new formula of fractional Taylor's series in Elzaki transform.

Proof: Consider the following fractional Taylor's series:

$$
\begin{aligned}
\Phi(\Upsilon)= & \aleph_{0}+\frac{\aleph_{1}}{\Gamma(\Phi+1)} \Upsilon^{\Phi}+\frac{\aleph_{2}}{\Gamma(2 \Phi+1)} \Upsilon^{2 \Phi} \\
& +\frac{\aleph_{3}}{\Gamma(3 \Phi+1)} \Upsilon^{3 \Phi}+\cdots .
\end{aligned}
$$

Applying Elzaki transform at the both sides of equation 


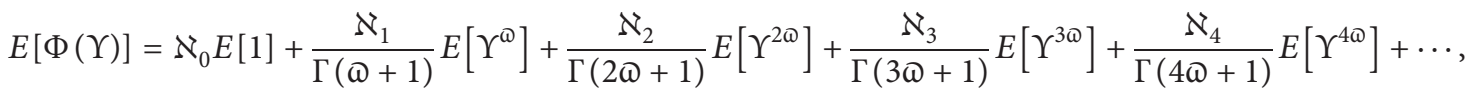

$$
\begin{aligned}
& \Psi(v)=\sum_{n=0}^{\infty} \aleph_{n} v^{n \varpi+2} .
\end{aligned}
$$

which is a new form of fractional Taylor formula in Elzaki transform form.

Remark 1. The multiple fractional Taylor's series or generalized form of Taylor's series representation at $\Upsilon=0$ takes the following form in Elzaki transform space:

$$
\begin{aligned}
\Psi(\chi, v) & =\sum_{n=0}^{\infty} \aleph_{n}(\chi) v^{n \varpi+2}, \\
\text { where } \chi & =\left(\chi_{1}, \chi_{2}, \chi_{3} \ldots \chi_{d}\right) \in \Re^{d}, d \in N .
\end{aligned}
$$

Lemma 2. Assume that the function $E[\Phi(\Upsilon)]=\Psi(v)$ has fractional power series (FPS) representation as follows:

$$
\Psi(v)=\sum_{n=0}^{\infty} \aleph_{n} v^{n \omega+2}
$$

Then, $\lim _{v \longrightarrow 0}\left(1 / v^{2}\right) \Psi(v)=\aleph_{0}$.

Proof: From the new form of fractional Taylor's series, we have

$$
\frac{1}{v^{2}} \Psi(v)=\aleph_{0}+\aleph_{1} v^{\Phi}+\aleph_{2} v^{2 \Phi}+\aleph_{3} v^{3 \oplus}+\aleph_{4} v^{4 \oplus}+\cdots
$$

Taking limit $v \longrightarrow 0$, so the last equation becomes as

$$
\lim _{v \longrightarrow 0} \frac{1}{v^{2}} \Psi(v)=\aleph_{0}=\Phi(0) .
$$

Remark 2. In the case of a generalized form of Taylor's series in Elzaki transform space, we have the following:

$$
\begin{aligned}
& \lim _{v \longrightarrow 0} \frac{1}{v^{2}} \Psi(\chi, v)=\aleph_{0}(\chi, 0), \\
& \text { where } \chi=\left(\chi_{1}, \chi_{2}, \chi_{3} \ldots \chi_{d}\right) \in \mathfrak{R}^{d}, d \in N .
\end{aligned}
$$

Lemma 3. Presume that $\Phi(\Upsilon)$ is a piecewise continuous function on $[0, \infty)$ and exponential order, $E[\Phi(\Upsilon)]=\Psi(v)$.

Then,

$$
E\left[D_{\Upsilon}^{n \oplus} \Phi(\Upsilon)\right]=\frac{E[\Phi(\Upsilon)]}{v^{n \oplus}}-\sum_{j=0}^{n-1} v^{(j-n) \oplus+2}\left(D_{\Upsilon}^{j \oplus} \Phi\right)(0),
$$

where $D_{\Upsilon}^{n \oplus} \Phi=D_{\Upsilon}^{\Phi} \cdot D_{\Upsilon}^{\Phi} \cdot D_{\Upsilon}^{\oplus} \ldots D_{\Upsilon}^{\Phi}(n-$ times $)$
Proof: To prove, we use the principle of mathematical induction method.

Using $n=1$ in equation (14),

$$
E\left[D_{\Upsilon}^{\oplus} \Phi(\Upsilon)\right]=\frac{\mathrm{E}[\Phi(\Upsilon)]}{v^{\Phi}}-v^{-\oplus+2} \Phi(0)
$$

Equation (14) is effective for $n=1$.

Using $n=2$ in equation (14), we get

$$
\begin{aligned}
& E\left[D_{\Upsilon}^{2 \omega} \Phi(\Upsilon)\right]=\frac{E[\Phi(\Upsilon)]}{v^{2 \Phi}}-v^{-2 \omega+2} \Phi(0)+v^{-\oplus+2}\left(D_{\Upsilon}^{\varpi} \Phi\right)(0), \\
& \text { L.H.S }=E\left[D_{\Upsilon}^{2 \oplus} \Phi(\Upsilon)\right]=E\left[D_{\Upsilon}^{\varpi}\left(D_{\Upsilon}^{\varpi} \Phi(\Upsilon)\right)\right] .
\end{aligned}
$$

Let $D_{\Upsilon}^{\oplus} \Phi(\Upsilon)=z(\Upsilon)$.

So, equation (16) becomes as

$$
\text { L.H.S }=E\left[D_{\Upsilon}^{2 \oplus} \Phi(\Upsilon)\right]=E\left[D_{\Upsilon}^{\varpi}(z(\Upsilon))\right] .
$$

By utilizing Caputo fractional derivative, the last equation becomes as

$$
\text { L.H.S }=E\left[D_{\Upsilon}^{2 \oplus} \Phi(\Upsilon)\right]=E\left[J_{\Upsilon}^{1-\oplus} z^{(1)}(\Upsilon)\right] .
$$

By using Riemann-Liouville integral formula of Elzaki transform,

$$
\text { L.H.S }=E\left[D_{\Upsilon}^{2 \oplus} \Phi(\Upsilon)\right]=v^{1-\oplus} E\left[z^{(1)}(\Upsilon)\right] .
$$

By differential property, the above equation becomes as follows:

$$
\begin{array}{r}
E\left[D_{\Upsilon}^{2 \oplus} \Phi(\Upsilon)\right]=v^{-\oplus} Z(v)-v^{2-\Phi} z(0) \\
\text { where }\left(D_{\Upsilon}^{\Phi} \Phi\right)(0)=z(0)
\end{array}
$$

From equation (20), we have

$$
\begin{aligned}
& E\left[D_{\Upsilon}^{2 \oplus} \Phi(\Upsilon)\right]=v^{-\oplus} E\left[D_{\Upsilon}^{\oplus} \Phi(\Upsilon)\right]-v^{2-\oplus}\left(D_{\Upsilon}^{\oplus} \Phi\right)(0) \\
& E\left[D_{\Upsilon}^{2 \oplus} \Phi(\Upsilon)\right]=\frac{E[\Phi(\Upsilon)]}{v^{2 Ф}}-v^{2-2 \Phi} \Phi(0)-v^{2-\oplus}\left(D_{\Upsilon}^{\oplus} \Phi\right)(0)
\end{aligned}
$$

So, from equation (21), we conclude that formula equation (14) is accurate when $n=2$. Now, suppose formula is valid for $n=r$. So, we have

$$
E\left[D_{\Upsilon}^{r \oplus} \Phi(\Upsilon)\right]=\frac{E[\Phi(\Upsilon)]}{v^{r \oplus}}-\sum_{j=0}^{r-1} v^{(j-r) \oplus+2}\left(D_{\Upsilon}^{j \oplus} \Phi\right)(0)
$$




$$
\begin{gathered}
E\left[D_{\Upsilon}^{(r+1) \oplus} \Phi(\Upsilon)\right]=\frac{E[\Phi(\Upsilon)]}{v^{r \oplus}}-\sum_{j=0}^{r-1+1} v^{(j-(r+1)) \oplus+2}\left(D_{\Upsilon}^{j \oplus} \Phi\right)(0), \\
\text { L.H.S }=E\left[D_{\Upsilon}^{(r+1) \oplus} \Phi(\Upsilon)\right], \\
\text { L.H.S }=E\left[D_{\Upsilon}^{\oplus}\left(D_{\Upsilon}^{r \oplus} \Phi(\Upsilon)\right)\right] .
\end{gathered}
$$

Suppose that

$$
D_{\Upsilon}^{r \oplus} \Phi(\Upsilon)=b(\Upsilon)
$$

So, equation (24) becomes as

$$
\text { L.H.S }=E\left[D_{\Upsilon}^{\oplus}(b(\Upsilon))\right] .
$$

By utilizing Caputo fractional derivative, so the last equation becomes as

$$
\text { L.H.S }=E\left[J_{\Upsilon}^{1-\oplus} b^{(1)}(\Upsilon)\right] .
$$

By utilizing Riemann-Liouville fractional integral formula, equation (27) becomes as follows:

$$
\text { L.H.S }=v^{1-\oplus} E\left[b^{(1)}(\Upsilon)\right] \text {. }
$$

By differential property of E-L, so equation (28) becomes as

$$
\text { L.H.S }=v^{\oplus} E\left[D_{\Upsilon}^{r \oplus} \Phi(\Upsilon)\right]-v^{2-\oplus} b(0),
$$

where $\left(D_{\Upsilon}^{r \oplus} \Phi\right)(0)=b(0)$.

From equation (29), we get

$$
\begin{aligned}
& \text { L.H.S }=\frac{E[\Phi(\Upsilon)]}{v^{(r+1) \oplus}}-\sum_{j=0}^{r-1} v^{(j-(r+1)) \oplus+2}\left(D_{\Upsilon}^{j \oplus} \Phi\right)(0)-v^{2-\Phi} b(0), \\
& \text { L.H.S }=\frac{E[\Phi(\Upsilon)]}{v^{n \varpi}}-\sum_{j=0}^{n-1} v^{(j-n) \oplus+2}\left(D_{\Upsilon}^{j \oplus} \Phi\right)(0) .
\end{aligned}
$$

So, equation (14) is valid for all integers. Thus, the proof completes.

Remark 3. By making generalization, the above proved formula takes the following form:

$$
E\left[D_{\Upsilon}^{n \oplus} \Phi(\chi, \Upsilon)\right]=\frac{E[\Phi(\chi, \Upsilon)]}{v^{n \oplus}}-\sum_{j=0}^{n-1} v^{(j-n) \oplus+2}\left(D_{\Upsilon}^{j \oplus} \Phi\right)(0), \quad \text { where } \chi=\left(\chi_{1}, \chi_{2}, \chi_{3}, \ldots \chi_{d}\right) \in \mathfrak{R}^{d}, d \in N
$$

and $D_{\Upsilon}^{n \oplus} \Phi=D_{\Upsilon}^{\Phi} \cdot D_{\Upsilon}^{\Phi} \cdot D_{\Upsilon}^{\oplus} \ldots D_{\Upsilon}^{\Phi}(n-$ times $)$.

Theorem 1. Suppose that the function $E[\Phi(\Upsilon)]=\Psi(v)$ has FPS representation as follows:

$$
\Psi(v)=\sum_{n=0}^{\infty} \aleph_{n} v^{n \varpi+2}
$$

then we have $\aleph_{n}=\left(D_{\Upsilon}^{n \omega} \Phi\right)(0)$, where $D_{\Upsilon}^{n \omega} \Phi=D_{\Upsilon}^{\varpi} \cdot D_{\Upsilon}^{\oplus} \cdot D_{\Upsilon}^{\oplus}$ $\ldots D_{\Upsilon}^{\oplus}(n-$ times $)$.

Proof: Consider new form of Taylor's series.

$$
\Psi(v)=\aleph_{0} v^{2}+\aleph_{1} v^{\Phi+2}+\aleph_{2} v^{2 \omega+2}+\aleph_{3} v^{3 \varpi+2}+\aleph_{4} v^{4 \varpi+2}+\cdots
$$

From the above equation, we have

$$
\begin{aligned}
& \aleph_{1}=\frac{1}{v^{\Phi+2}} \Psi(v)-\frac{1}{v^{\Phi+2}} v^{2} \Psi(0)-\frac{1}{v^{\Phi+2}} \aleph_{2} v^{2 \omega+2} \\
& -\frac{1}{v^{\oplus+2}} \aleph_{3} v^{3 \omega+2}-\frac{1}{v^{\Phi+2}} \aleph_{4} v^{4 \omega+2}+\cdots .
\end{aligned}
$$

Taking $v \longrightarrow 0$ on the above equation,

$$
\aleph_{1}=\lim _{v \longrightarrow 0} \frac{1}{v^{2}}\left(\frac{1}{v^{\Phi}} \Psi(v)-\frac{1}{v^{\Phi-2}} \Psi(0)\right)
$$

By using Lemma 3,

$$
\aleph_{1}=\lim _{v \longrightarrow 0} \frac{1}{v^{2}}\left(E\left[D_{\Upsilon}^{\oplus} \Phi(\Upsilon)\right](v)\right)
$$

By Lemma 2, the above equation becomes as

$$
\aleph_{1}=\left(D_{\Upsilon}^{\oplus} \Phi\right)(0)
$$

Again from equation (32),

$$
\begin{aligned}
\aleph_{2}= & \frac{1}{v^{2}}\left(\frac{1}{v^{2 \Phi}} \Psi(v)-\frac{1}{v^{2 \Phi-2}} \Psi(0)-\frac{1}{v^{\Phi-2}} \aleph_{1}\right)-\aleph_{3} v^{\Phi} \\
& -\aleph_{4} v^{2 \Phi}+\cdots .
\end{aligned}
$$

Taking $v \longrightarrow 0$ on the last equation, utilizing Lemma 3, the above equation becomes as

$$
\aleph_{2}=\lim _{v \longrightarrow 0} \frac{1}{v^{2}}\left(E\left[D_{\Upsilon}^{2 \oplus} \Phi(\Upsilon)\right](v)\right) .
$$

By Lemma 2,

$$
\aleph_{2}=\left(D_{\Upsilon}^{2 \oplus} \Phi\right)(0)
$$

Again from equation (32), we have

$$
\aleph_{3}=\lim _{v \longrightarrow 0} \frac{1}{v^{2}}\left(\frac{1}{v^{3 \omega}} \Psi(v)-\Psi(0) \frac{1}{v^{3 \omega-2}}-\aleph_{1} \frac{1}{v^{2 \Phi}}-\aleph_{2} \frac{1}{v^{\Phi-2}}\right) .
$$

By Lemma 3, 


$$
\aleph_{3}=\lim _{v \longrightarrow 0}\left(\frac{1}{v^{2}} E\left[D_{\Upsilon}^{3 \oplus} \Phi(\Upsilon)\right](v)\right)
$$

By Lemma 2, the last equation becomes as

$$
\aleph_{3}=\left(D_{\Upsilon}^{3 \oplus} \Phi\right)(0) \text {. }
$$

In the same manner, we can obtain the following form by making generalization:

$$
\aleph_{n}=\left(D_{\Upsilon}^{n \oplus} \Phi\right)(0)
$$

This completes the proof of the theorem.

Remark 4. For multiple Taylor's series, the proved result becomes as follows:

$\aleph_{n}(\chi)=\left(D_{\Upsilon}^{n \omega} \Phi\right)(0), \quad$ where $\chi=\left(\chi_{1}, \chi_{2}, \chi_{3}, \ldots \chi_{d}\right) \in \mathfrak{R}^{d}, d \in N$.

The following theorem describes and determines the conditions for convergence of the new form of Taylor's formula that are introduced in Lemma 1.

Theorem 2. Let $\Psi(v)=E[\Phi(Y)]$ be represented as the new form of fractional Taylor's formula as in Elzaki transform:

$$
\Psi(v)=\sum_{n=0}^{\infty} \aleph_{n} v^{n \varpi+2}
$$

if

$$
\left|\frac{1}{v^{2}} E\left[D_{\Upsilon}^{(n+1) \oplus} \Phi(\Upsilon)\right]\right| \leq T
$$

Then, the remainder $R_{n}(v)$ of the new form of fractional Taylor's formula satisfies the following inequality:

$$
\left|R_{n}(v)\right| \leq v^{(n+1) \omega+2} T .
$$

Proof: Consider the following:

$\Psi_{n}(v)=\aleph_{0} v^{2}+\aleph_{1} v^{\Phi+2}+\aleph_{2} v^{2 \omega+2}+\aleph_{3} v^{3 \omega+2}+\cdots \aleph_{n} v^{n \varpi+2}$.

From equations (46) and (49), we get

$$
R_{n}(v)=\Psi(v)-\sum_{k=0}^{n} \aleph_{k} v^{k \varpi+2}
$$

By Theorem 1,

$$
\begin{gathered}
R_{n}(v)=\Psi(v)-\sum_{k=0}^{n} v^{k \oplus+2}\left(D_{\Upsilon}^{k \oplus} \Phi\right)(0) \\
\frac{1}{v^{(n+1) \oplus+2}} R_{n}(v)=\frac{1}{v^{2}}\left(\frac{1}{v^{(n+1) \Phi}} \Psi(v)-\sum_{k=0}^{n} \frac{1}{v^{(n+1-k) \oplus-2}}\left(D_{\Upsilon}^{k \oplus} \Phi\right)(0)\right) .
\end{gathered}
$$

By Lemma 3,

$$
\begin{gathered}
\frac{1}{v^{(n+1) \oplus+2}} R_{n}(v)=\frac{1}{v^{2}} E\left[D_{\Upsilon}^{(n+1) \oplus} \Phi(\Upsilon)\right], \\
\left|\frac{1}{v^{(n+1) \oplus+2}} R_{n}(v)\right|=\left|\frac{1}{v^{2}} E\left[D_{\Upsilon}^{(n+1) \oplus} \Phi(\Upsilon)\right]\right| .
\end{gathered}
$$

By the given assumption, the above equation becomes as

$$
-v^{(n+1) \oplus+2} T \leq R_{n}(v) \leq v^{(n+1) \oplus+2} T,
$$

$\left|R_{n}(v)\right| \leq v^{(n+1) \oplus+2} T$. Hence, the required result is proved.

\section{Demonstrating the ERPSM for the CRDEs}

We exploit our novel ERPSM to originate the results of the linear and nonlinear CRDEs. The foremost set of rules of this method for resolving the CRDEs can be accumulated by the following steps: employing the Elzaki transform to CRDE and then deploying the novel form of Taylor's series to introduce the solution of CRDE in the novel space. The coefficients of this series are established with a new idea. At the end, employing the inverse Elzaki transform to achieve the solution of the problem in the actual space.

3.1. Elzaki Residual Power Series Solutions for the CRDEs. In this subsection, we systematized the stages for conquering the Elzaki residual power series solution for the linear and nonlinear CRDE by the following procedure.

Step 1. Rewriting equation (2) as demonstrated:

$$
\frac{\partial^{\oplus} \Phi(\chi, \Upsilon)}{\partial \Upsilon^{\Phi}}-\lambda \frac{\partial^{2} \Phi(\chi, \Upsilon)}{\partial \chi^{2}}-z(\chi, \Upsilon) \Phi(\chi, \Upsilon)=0
$$

Step 2. Manipulating Elzaki transform at both sides of equation (54), we get in this way

$$
E\left[\frac{\partial^{\oplus} \Phi(\chi, \Upsilon)}{\partial \Upsilon^{\Phi}}\right]-\lambda E\left[\frac{\partial^{2} \Phi(\chi, \Upsilon)}{\partial \chi^{2}}\right]-E\left[E^{-1}[Z(\chi, v)] E^{-1}[\Psi(\chi, v)]\right]=0
$$


where

$$
\begin{aligned}
E^{-1}[Z(\chi, v)] & =z(\chi, \Upsilon), \\
E^{-1}[\Psi(\chi, v)] & =\Phi(\chi, \Upsilon), \\
E\left[\frac{\partial^{\oplus} \Phi(\chi, \Upsilon)}{\partial \Upsilon^{Ф}}\right] & =\frac{\Psi(\chi, v)}{v^{\Phi}}-v^{-\oplus+2} g(\chi) .
\end{aligned}
$$

So, we get the following form:

$$
\begin{aligned}
\Psi(\chi, v)= & v^{2} g(\chi)+\lambda v^{\Phi} D_{\chi \chi} \Psi(\chi, v) \\
& +v^{\Phi} E\left[E^{-1}[Z(\chi, v)] E^{-1}[\Psi(\chi, v)]\right] .
\end{aligned}
$$

Step 3. Considering the solution of equation (57) as the following:

$$
\Psi(\chi, v)=\sum_{n=0}^{\infty} \aleph_{n}(\chi) v^{2+n \varpi}
$$

Step 4. Setting $\aleph_{0}(\chi)=\lim _{\nu \rightarrow \infty}\left(1 / v^{2}\right) \Psi(\chi, v)=\Phi(\chi, 0)$. Step 5. Establishing the $k$ th-truncated series of $\Psi(\chi, v)$ as

$$
\begin{aligned}
\Psi_{k}(\chi, v) & =\sum_{n=0}^{k} \aleph_{n}(\chi) v^{2+n \varpi}, \\
\aleph_{0} & =\lim _{v \longrightarrow 0} \frac{1}{v^{2}} \Psi(\chi, v), \\
\Psi_{k}(\chi, v) & =\aleph_{0} v^{2}+\sum_{n=1}^{k} \aleph_{n}(\chi) v^{2+n \varpi} .
\end{aligned}
$$

Step 6. Considering the Elzaki residual function (ERF) of equation (57) and the $k$ th-truncated ERF separately such that

$$
\begin{aligned}
\operatorname{ERes}(\chi, v)= & \Psi(\chi, v)-v^{2} g(\chi)-\lambda v^{\Phi} D_{\chi \chi} \Psi(\chi, v) \\
& -v^{\Phi} E\left[E^{-1}[Z(\chi, v)] E^{-1}[\Psi(\chi, v)]\right], \\
\operatorname{ERes}_{k}(\chi, v)= & \Psi_{k}(\chi, v)-v^{2} g(\chi)-\lambda v^{\Phi} D_{\chi \chi} \Psi_{k}(\chi, v) \\
& -v^{\Phi} E\left[E^{-1}[Z(\chi, v)] E^{-1}\left[\Psi_{k}(\chi, v)\right]\right] .
\end{aligned}
$$

Step 7. Replacing the series form of $\Psi_{k}(\chi, v)$ into equation (60).

Step 8. Dividing at both sides of equation (60) with $v^{k v+2}$ as follows:

$$
\begin{aligned}
\frac{1}{v^{2+k \omega}} \operatorname{ERes}_{k}(\chi, v)= & \frac{1}{v^{2+k \omega}} \Psi_{k}(\chi, v)-\frac{1}{v^{2+k \omega}} v^{2} g(\chi) \\
& -\lambda \frac{1}{v^{2+k \omega}} v^{\Phi} D_{\chi \chi} \Psi_{k}(\chi, v) \\
& -\frac{1}{v^{2+k \omega}} v^{\Phi} E\left[E^{-1}[Z(\chi, v)] E^{-1}\left[\Psi_{k}(\chi, v)\right]\right] .
\end{aligned}
$$

Step 9. Taking limit at both sides of equation (61).

$$
\begin{aligned}
\lim _{v \longrightarrow 0} \frac{1}{v^{2+k \omega}} \operatorname{ERes}_{k}(\chi, v)= & \lim _{v \longrightarrow 0} \frac{1}{v^{2+k \omega}} \Psi_{k}(\chi, v)-\lim _{v \longrightarrow 0} \frac{1}{v^{2+k \omega}} v^{2} g(\chi) \\
& -\lambda \lim _{v \longrightarrow 0} \frac{1}{v^{2+k \omega}} v^{\Phi} D_{\chi \chi} \Psi_{k}(\chi, v)-\lim _{v \longrightarrow 0} \frac{1}{v^{2+k \omega}} v^{\Phi} E\left[E^{-1}[Z(\chi, v)] E^{-1}\left[\Psi_{k}(\chi, v)\right]\right] .
\end{aligned}
$$

Step 10. Solving the following equation for $\aleph_{n}(\chi)$ :

$$
\lim _{v \longrightarrow 0}\left(\frac{1}{v^{k \oplus+2}} \operatorname{ERes}_{k}(\chi, v)\right)=0, \quad k=1,2,3, \ldots
$$

Step 11. Replacing the attained values of $\aleph_{n}(\chi)$ into $k$ th-truncated series of $\Psi(\chi, v)$ to get the $k$ th-approximate solution of equation (57).

Step 12. Manipulating the inverse Elzaki transform on $\Psi_{k}(\chi, v)$ to attain the $k$ th-approximate solution of $\Phi_{k}(\chi, \Upsilon)$ in the real space.
3.2. Applications to Linear and Nonlinear CRDEs. In this subsection, we consider three main problems of CRDEs to illustrate the execution and capability of ERPSM.

3.2.1. Approximate and Closed Form Solutions of Linear CRDEs. Two applications are considered for linear CRDEs.

Problem 1. Consider the time-fractional linear CRDE [19]. $D_{\Upsilon}^{\varpi} \Phi(\chi, \Upsilon)=\Phi_{\chi \chi}(\chi, \Upsilon)-\Phi(\chi, \Upsilon), \quad \chi, \Upsilon \geq 0,0<\bowtie \leq 1$. 
Subject to initial condition,

$$
\Phi(\chi, 0)=e^{-\chi}+\chi
$$

Solution. Utilizing Elzaki transform on equation (64),

$$
E\left[D_{\Upsilon}^{\oplus} \Phi(\chi, \Upsilon)\right]=E\left[\Phi_{\chi \chi}(\chi, \Upsilon)\right]-E[\Phi(\chi, \Upsilon)]
$$

where $E[\Phi(\chi, \Upsilon)]=\Psi(\chi, v)$,

$$
E\left[D_{\Upsilon}^{\oplus} \Phi(\chi, \Upsilon)\right]=\frac{\Psi(\chi, v)}{v^{\varpi}}-v^{-\oplus+2} \Phi(\chi, 0),
$$

so equation (66) becomes as

$$
\Psi(\chi, v)=v^{2} \Phi(\chi, 0)+v^{\Phi} D_{\chi \chi} \Psi(\chi, v)-v^{\Phi} \Psi(\chi, v) .
$$

Initiate a series solution to the algebraic equation (68). Hence, presume that the expansion of $\Psi(\chi, v)$ is the following:

$$
\Psi(\chi, v)=\sum_{n=0}^{\infty} \aleph_{n}(\chi) v^{2+n \omega}
$$

Assume that $\Psi(\chi, v)$ has the $k$ th-truncated series as

$$
\Psi_{k}(\chi, v)=\sum_{n=0}^{k} \aleph_{n}(\chi) v^{2+n \omega}
$$

By Lemma 2, we have

$$
\lim _{v \longrightarrow 0} \frac{1}{v^{2}} \Psi(\chi, v)=\Phi(\chi, 0)=e^{-\chi}+\chi .
$$

The $k$ th-truncated series becomes as follows:

$$
\Psi_{k}(\chi, v)=v^{2}\left(e^{-\chi}+\chi\right)+\sum_{n=1}^{k} \aleph_{n}(\chi) v^{2+n \omega} .
$$

The ERF of the algebraic equation (68) is described as

$$
\operatorname{ERes}(\chi, v)=\Psi(\chi, v)-v^{2}\left(e^{-\chi}+\chi\right)-v^{\Phi} D_{\chi \chi} \Psi(\chi, v)+v^{\Phi} \Psi(\chi, v) .
$$

Furthermore, $k$ th-truncated ERF of the algebraic equation (69) is explained as follows:

$$
\begin{aligned}
\operatorname{ERes}_{k}(\chi, v)= & \Psi_{k}(\chi, v)-v^{2}\left(e^{-\chi}+\chi\right) \\
& -v^{\oplus} D_{\chi \chi} \Psi_{k}(\chi, v)+v^{\oplus} \Psi_{k}(\chi, v) .
\end{aligned}
$$

By utilizing equations (72) and (74), we get undefined coefficients in the following form:

$$
\begin{aligned}
& \aleph_{1}(\chi)=-\chi, \\
& \aleph_{2}(\chi)=\chi, \\
& \aleph_{3}(\chi)=-\chi, \\
& \aleph_{4}(\chi)=\chi, \\
& \aleph_{5}(\chi)=-\chi .
\end{aligned}
$$

So, we get the $5^{\text {th }}$ approximate solution of Elzaki transform of equation (68).

$$
\Psi_{5}(\chi, v)=\frac{e^{-\chi}+\chi}{v^{2}}-\frac{\chi}{v^{2+\Phi}}+\frac{\chi}{v^{2+2 \omega}}-\frac{\chi}{v^{2+3 \omega}}+\frac{\chi}{v^{2+4 \omega}}-\frac{\chi}{v^{2+5 \omega}} .
$$

Operating inverse Elzaki transform on both sides of equation (76), we get the $5^{\text {th }}$ approximate solution of equation (64).

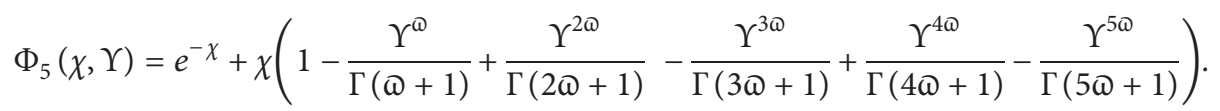

When $\Phi=1$, equation (77) becomes as

$$
\Phi_{5}(\chi, \Upsilon)=e^{-\chi}+\chi\left(1-\frac{\Upsilon}{1 !}+\frac{\Upsilon^{2}}{2 !}-\frac{\Upsilon^{3}}{3 !}+\frac{\Upsilon^{4}}{4 !}-\frac{\Upsilon^{5}}{5 !}\right)
$$

Equation (78) is coinciding with the six terms of the expansion of the exact solution $\Phi(\chi, \Upsilon)=e^{-\chi}+\chi e^{-\Upsilon}$.

Table 1 demonstrates the values of absolute error of the $5^{\text {th }}$ order approximate and exact solutions at $\Phi=1$ when $\chi=1$ which support the capability and exactness of the novel technique.

Figure 1 displays the evaluations of exact solution at $\Phi=$ 1 and the $5^{\text {th }}$ approximate solution of Problem 1 , at $\chi=1$, for several values of $\Upsilon$ and $\Phi$. Figure 1 confirms that when values of $₫$ approach to " 1 ," the approximate solution approaches to the exact solution, which approves the efficacy and correctness of the new method. Moreover, the approximate solution overlaps with the exact solution at $\Phi=1$ and this once more ratifies the usefulness and correctness of the ERPSM.

Problem 2. Consider the time-fractional linear CRDE [20],

$$
\begin{gathered}
D_{\Upsilon}^{\oplus} \Phi(\chi, \Upsilon)=\Phi_{\chi \chi}(\chi, \Upsilon)-\left(1+4 \chi^{2}\right) \Phi(\chi, \Upsilon) \\
\chi, \Upsilon \geq 0,0<@ \leq 1 .
\end{gathered}
$$

With the initial condition,

$$
\Phi(\chi, 0)=e^{\chi^{2}} .
$$
(79), 
TABLE 1: Absolute error of ERPS results.

\begin{tabular}{lccc}
\hline$\Upsilon$ & Exact solution & Approximate solution & Absolute error \\
\hline 0 & 2.71828182846 & 2.71828182846 & 0 \\
0.06 & 2.88637098927 & 2.88637098909 & $1.7766543792 \times 10-10$ \\
0.12 & 3.06485420329 & 3.06485419182 & $1.1469452055 \times 10-8$ \\
0.18 & 3.25437420289 & 3.2543740711 & $1.3178722424 \times 10-7$ \\
0.24 & 3.45561346476 & 3.45561271778 & $7.4698766506 \times 10-7$ \\
0.30 & 3.66929666762 & 3.66929379283 & 0.00000287478963168 \\
0.36 & 3.8961933018 & 3.89618464113 & 0.00000866066429372 \\
0.42 & 4.13712044025 & 4.13709840516 & 0.0000220350955411 \\
0.48 & 4.39294568092 & 4.39289613873 & 0.044093686996 \\
\hline
\end{tabular}

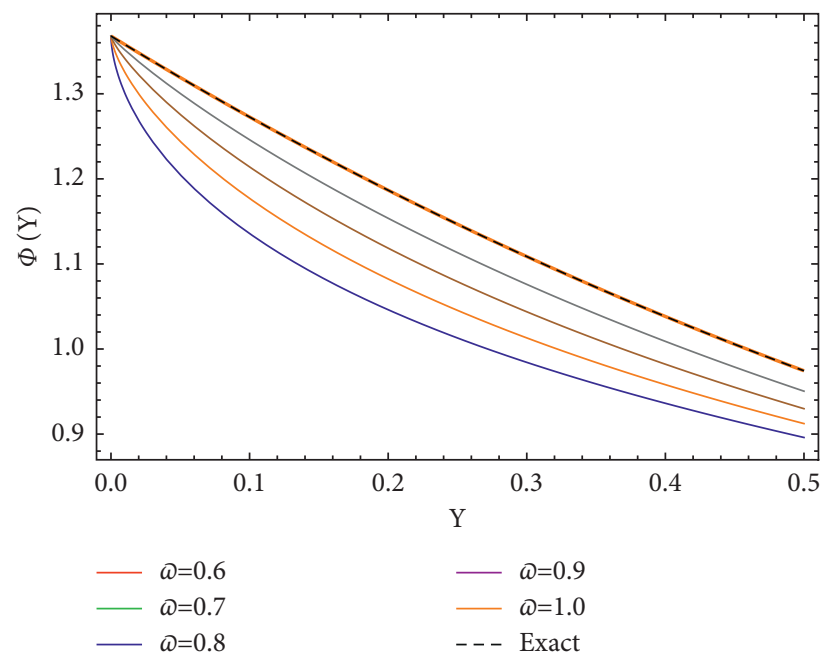

FIgURE 1: Evaluation of closed form and approximate consequences of Problem 1.

$$
\begin{aligned}
E\left[D_{\Upsilon}^{\Phi} \Phi(\chi, \Upsilon)\right] & =E\left[\Phi_{\chi \chi}(\chi, \Upsilon)\right]-\left(1+4 \chi^{2}\right) E[\Phi(\chi, \Upsilon)], \\
\Psi(\chi, v) & =v^{2} e^{\chi^{2}}+v^{\Phi} D_{\chi \chi} \Psi(\chi, v)-v^{\Phi}\left(1+4 \chi^{2}\right) \Psi(\chi, v),
\end{aligned}
$$

where

$$
\begin{gathered}
E\left[D_{\Upsilon}^{\varpi} \Phi(\chi, \Upsilon)\right]=\frac{\Psi(\chi, v)}{v^{\varpi}}-v^{-\oplus+2} e^{\chi^{2}}, \\
E[\Phi(\chi, \Upsilon)]=\Psi(\chi, v) .
\end{gathered}
$$

Now, establishing a series solution of equation (81), consequently assume that $\Psi(\chi, v)$ has the expansion as follows:

$$
\Psi(\chi, v)=\sum_{n=0}^{\infty} \aleph_{n}(\chi) v^{2+n \omega}
$$

The $k$ th-truncated series $\Psi(\chi, v)$ is as follows:

$$
\Psi_{k}(\chi, v)=\sum_{n=0}^{k} \aleph_{n}(\chi) v^{2+n \omega} .
$$

By Lemma 2, we have

$$
\lim _{v \longrightarrow 0} \frac{1}{v^{2}} \Psi(\chi, v)=\Phi(\chi, 0)=e^{\chi^{2}}
$$

So, equation (84) becomes as

$$
\Psi_{k}(\chi, v)=e^{\chi^{2}} v^{2}+\sum_{n=1}^{k} \aleph_{n}(\chi) v^{2+n \omega} .
$$

The ERF of equation (81) is defined as

$$
\begin{aligned}
\operatorname{ERes}(\chi, v)= & \Psi(\chi, v)-v^{2} e^{\chi^{2}}-v^{\oplus} D_{\chi \chi} \Psi(\chi, v) \\
& +v^{\oplus}\left(1+4 \chi^{2}\right) \Psi(\chi, v) .
\end{aligned}
$$

The $k$ th-ERF is as follows:

$$
\begin{aligned}
\operatorname{ERes}_{k}(\chi, v)= & \Psi_{k}(\chi, v)-v^{2} e^{\chi^{2}}-v^{\oplus} D_{\chi \chi} \Psi_{k}(\chi, v) \\
& +v^{\oplus}\left(1+4 \chi^{2}\right) \Psi_{k}(\chi, v) .
\end{aligned}
$$

To find unspecified coefficients using equations (86) and (88), so we have

$$
\begin{aligned}
& \aleph_{1}(\chi)=e^{\chi^{2}} \\
& \aleph_{2}(\chi)=e^{\chi^{2}}, \\
& \aleph_{3}(\chi)=e^{\chi^{2}}, \\
& \aleph_{4}(\chi)=e^{\chi^{2}}, \\
& \aleph_{5}(\chi)=e^{\chi^{2}}
\end{aligned}
$$


The $5^{\text {th }}$ approximate solution of equation (81) in Elzaki transform form is

$$
\Psi_{5}(\chi, v)=e^{\chi^{2}}\left(\frac{1}{v^{2}}+\frac{1}{v^{2+\Phi}}+\frac{1}{v^{2+2 \Phi}}+\frac{1}{v^{2+3 \Phi}}+\frac{1}{v^{2+4 \omega}}+\frac{1}{v^{2+5 \Phi}}\right) .
$$

By applying inverse Elzaki transform on equation (90), we get the $5^{\text {th }}$ approximate solution of equation (79) as follows:

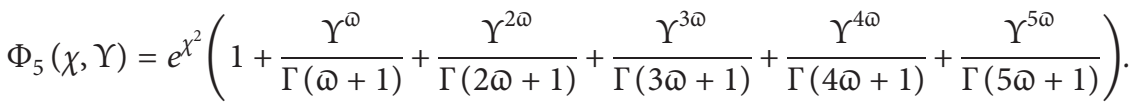

When $\Phi=1$, equation (91) becomes as

$$
\Phi_{5}(\chi, \Upsilon)=e^{\chi^{2}}\left(1+\frac{\Upsilon}{1 !}+\frac{\Upsilon^{2}}{2 !}+\frac{\Upsilon^{3}}{3 !}+\frac{\Upsilon^{4}}{4 !}+\frac{\Upsilon^{5}}{5 !}\right)
$$

Equation (92) represents the first six terms of the expansion of $e^{\chi^{2}+Y}$, so closed form solution of equation (79) is $e^{\chi^{2}+\Upsilon}$.

Table 2 demonstrates the values of absolute error of the $5^{\text {th }}$ order approximate and exact solutions at $\omega=1$ when $\chi=1$, which support the capability and accuracy of the new technique.

Figure 2 demonstrates the exploits of exact solution at $\Phi=1$ and the $5^{\text {th }}$ approximate solution of Problem 2, when $\chi=1$ for numerous values of $\Upsilon$ and $\omega$. The figure endorses that when values of $₫$ approach to " 1 ," the approximate solution approaches to exact solution, which supports the ability and precision of the new method. Moreover, the approximate solution overlaps with the exact solution at $₫=$ 1 and this once more ratifies the usefulness and correctness of the ERPSM.

\subsubsection{Approximate and Closed Form Solutions of Nonlinear CRDES}

Problem 3. Consider the nonlinear time-fractional CRDE [21],

$$
\begin{aligned}
D_{\Upsilon}^{\oplus} \Phi(\chi, \Upsilon)= & \Phi_{\chi \chi}(\chi, \Upsilon)-\Phi_{\chi}(\chi, \Upsilon)+\Phi(\chi, \Upsilon) \Phi_{\chi \chi}(\chi, \Upsilon) \\
& -\Phi^{2}(\chi, \Upsilon)+\Phi(\chi, \Upsilon), \quad \chi, \Upsilon \geq 0,0<® \leq 1
\end{aligned}
$$

With the initial condition,

$$
\Phi(\chi, 0)=e^{\chi}
$$

Solution. By applying Elzaki transform on equation (93), we get

$$
\begin{aligned}
\Psi(\chi, v)= & v^{2} e^{\chi}+v^{\Phi} D_{\chi \chi} \Psi(\chi, v)-v^{\oplus} D_{\chi} \Psi(\chi, v) \\
& +v^{\Phi} E\left[E^{-1}[\Psi(\chi, v)] D_{\chi \chi} E^{-1}[\Psi(\chi, v)]\right] \\
& -v^{\Phi} E\left[\left[E^{-1} \Psi(\chi, v)\right]^{2}\right]+v^{\oplus} \Psi(\chi, v) .
\end{aligned}
$$

Here,

$$
\begin{aligned}
E\left[\Phi_{\chi \chi}(\chi, \Upsilon)\right] & =D_{\chi \chi} \Psi(\chi, v), \\
E\left[\Phi_{\chi}(\chi, \Upsilon)\right] & =D_{\chi} \Psi(\chi, v), \\
\Phi(\chi, \Upsilon) & =E^{-1}[\Psi(\chi, v)], \\
\Phi^{2}(\chi, \Upsilon) & =\left[E^{-1}[\Psi(\chi, v)]\right]^{2}, \\
\Phi_{\chi \chi}(\chi, \Upsilon) & =D_{\chi \chi} E^{-1}[\Psi(\chi, v)], \\
E\left[D_{\Upsilon}^{\oplus} \Phi(\chi, \Upsilon)\right] & =\frac{\Psi(\chi, v)}{v^{\Phi}}-v^{-\oplus+2} e^{\chi}
\end{aligned}
$$

Define a series solution of equation (95) as follows:

$$
\Psi(\chi, v)=\sum_{n=0}^{\infty} \aleph_{n}(\chi) v^{2+n \varpi}
$$

The $k$ th-truncated series is

$$
\Psi_{k}(\chi, v)=\sum_{n=0}^{k} \aleph_{n}(\chi) v^{2+n ळ}
$$

By Lemma 2,

$$
\lim _{v \longrightarrow 0} \frac{1}{v^{2}} \Psi(\chi, v)=\Phi(\chi, 0)=e^{\chi} .
$$

Therefore, the $k$ th-truncated series becomes as

$$
\Psi_{k}(\chi, v)=e^{\chi} v^{2}+\sum_{n=1}^{k} \aleph_{n}(\chi) v^{2+n \omega} .
$$

Now, define ERF in the following form:

$$
\begin{aligned}
\operatorname{ERes}(\chi, v)= & \Psi(\chi, v)-v^{2} e^{\chi}-v^{\Phi} D_{\chi \chi} \Psi(\chi, v)+v^{\Phi} D_{\chi} \Psi(\chi, v) \\
& -v^{\Phi} E\left[E^{-1}[\Psi(\chi, v)] D_{\chi \chi} E^{-1}[\Psi(\chi, v)]\right] \\
& +v^{\Phi} E\left[\left[E^{-1} \Psi(\chi, v)\right]^{2}\right]-v^{\Phi} \Psi(\chi, v) .
\end{aligned}
$$

The $k$ th-truncated ERF is 
TABLE 2: Absolute error of ERPS results.

\begin{tabular}{lccc}
\hline$\Upsilon$ & Exact solution & Approximate solution & Absolute error \\
\hline 0.00 & 1.36787944117 & 1.36787944117 & 0.0 \\
0.06 & 1.30964397476 & 1.30964397469 & $7 \times 10-11$ \\
0.12 & 1.25479987789 & 1.25479987381 & $4.08 \times 10-9$ \\
0.18 & 1.20314965258 & 1.20314960653 & $4.605 \times 10-8$ \\
0.24 & 1.15450730224 & 1.15450704565 & $2.5659 \times 10-7$ \\
0.30 & 1.10869766185 & 1.10869669117 & $9.7068 \times 10-7$ \\
0.36 & 1.06555576724 & 1.06555289269 & 0.00000287455 \\
0.42 & 1.02492626099 & 1.02491907181 & 0.00000718918 \\
0.48 & 0.98666283297 & 0.98664694453 & 0.000015888447 \\
\hline
\end{tabular}

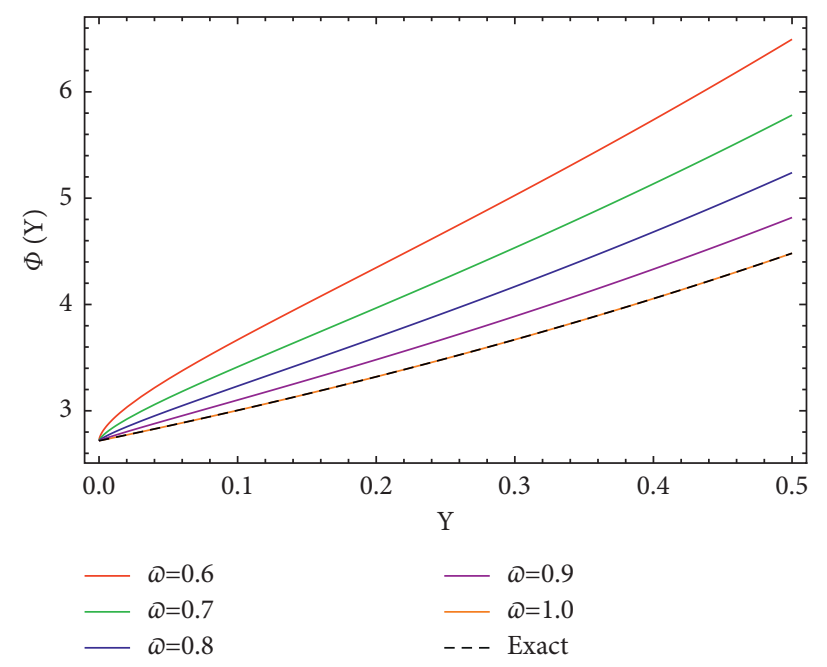

Figure 2: The behavior of exact and approximate outcomes of Problem 2.

$$
\begin{aligned}
\operatorname{ERes}_{k}(\chi, v)= & \Psi_{k}(\chi, v)-v^{2} e^{\chi}-v^{\oplus} D_{\chi \chi} \Psi_{k}(\chi, v) \\
& +v^{\Phi} D_{\chi} \Psi_{k}(\chi, v) \\
& -v^{\Phi} E\left[E^{-1}\left[\Psi_{k}(\chi, v)\right] D_{\chi \chi} E^{-1}\left[\Psi_{k}(\chi, v)\right]\right] \\
& +v^{\Phi} E\left[\left[E^{-1} \Psi_{k}(\chi, v)\right]^{2}\right]-v^{\oplus} \Psi_{k}(\chi, v) .
\end{aligned}
$$

The undefined coefficients are determined in the following form by utilizing equations (100) and (102).

$$
\begin{aligned}
& \aleph_{1}(\chi)=e^{\chi}, \\
& \aleph_{2}(\chi)=e^{\chi}, \\
& \aleph_{3}(\chi)=e^{\chi}, \\
& \aleph_{4}(\chi)=e^{\chi}, \\
& \aleph_{5}(\chi)=e^{\chi} .
\end{aligned}
$$

The $5^{\text {th }}$ approximate solution of equation (95) is given as

$$
\Psi_{5}(\chi, v)=e^{\chi}\left(\frac{1}{v^{2}}+\frac{1}{v^{2+\Phi}}+\frac{1}{v^{2+2 \Phi}}+\frac{1}{v^{2+3 \omega}}+\frac{1}{v^{2+4 \Phi}}+\frac{1}{v^{2+5 \Phi}}\right) .
$$

By applying inverse Elzaki transform on the above equation, we get the $5^{\text {th }}$ approximate solution of equation (93).

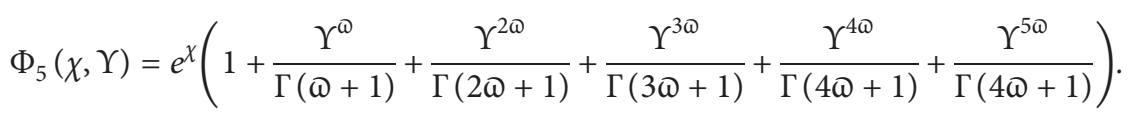

For $\Phi=1$, the last equation becomes as 
TABLE 3: Absolute error of ERPS results.

\begin{tabular}{lccc}
\hline$\Upsilon$ & Exact solution & Approximate solution & Absolute error \\
\hline 0.00 & 1.36787944117 & 1.36787944117 & 0.0 \\
0.06 & 1.30964397476 & 1.30964397469 & $7 \times 10-11$ \\
0.12 & 1.25479987789 & 1.25479987381 & $4.08 \times 10-9$ \\
0.18 & 1.20314965258 & 1.20314960653 & $4.605 \times 10-8$ \\
0.24 & 1.15450730224 & 1.15450704565 & $2.5659 \times 10-7$ \\
0.30 & 1.10869766185 & 1.10869669117 & $9.7068 \times 10-7$ \\
0.36 & 1.06555576724 & 1.06555289269 & 0.00000287455 \\
0.42 & 1.02492626099 & 1.02491907181 & 0.00000718918 \\
0.48 & 0.98666283297 & 0.98664694453 & 0.000015888447 \\
\hline
\end{tabular}

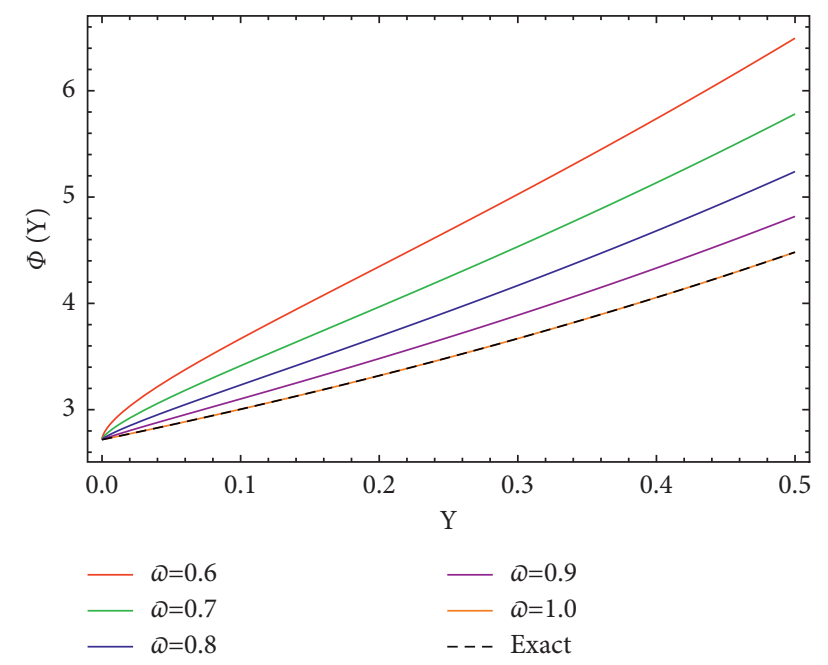

Figure 3: 2D plot of exact and approximate solution of Problem 3.

$$
\Phi_{5}(\chi, \Upsilon)=e^{\chi}\left(1+\frac{\Upsilon}{1 !}+\frac{\Upsilon^{2}}{2 !}+\frac{\Upsilon^{3}}{3 !}+\frac{\Upsilon^{4}}{4 !}+\frac{\Upsilon^{5}}{5 !}\right)
$$

Equation (106) coincides with the $1^{\text {st }}$ six terms of the expansion of $e^{\chi+\Upsilon}$, therefore exact solution of equation (93) is $e^{\chi+\Upsilon}$.

Table 3 demonstrates the values of absolute error of the $5^{\text {th }}$ order approximate and exact results at $\omega=1$, and $\chi=1$, which support the ability and accuracy of the novel technique.

Figure 3 establishes the actions of exact solution at $₫=1$ and the $5^{\text {th }}$ approximate solution of Problem 3 , when $\chi=1$ for certain values of $\Upsilon$ and $\varpi$. The figure recommends that when values of $₫$ approach to " 1 ," the approximate solution approaches to exact solution, which supports the capability and exactness of the new method. Moreover, the approximate solution overlaps with the exact solution at $\Phi=1$ and this once more ratifies the usefulness and correctness of the ERPSM.

\section{Conclusions}

There are enormous number of numerical and analytical methods for resolving the DEs; there are numerous methods that have superiority over the others. Few of them are precise and operative, but they necessitate mathematical operations that can be problematic and elongated. Our novel method,
ERPSM, is considered by accurateness, rapidity, and effortlessness in finding exact and approximate solutions to DEs.

To study the efficiency and reliability of ERPSM for PDEs, absolute errors of three applications are scrutinized. Consequences verify that our novel technique is simple, accurate, applicable, and efficient. The recommended techniques offered us an effortless and quick technique to perceive the coefficients of the suggested series to be a solution to the equation. Dissimilar to the traditional RPS method, while establishing the coefficients for a series, it is required to compute the fractional derivative every time, while ERPSM only requires the concept of the limit at zero in establishing the coefficients for the series.

The gain of the ERPSM is that it decreases considerably the numerical calculations to construct the consequences for this category of equations related to existing methods, for instance, the differential transform method (DTM), perturbation method, and Adomian decomposition method (ADM). Consequently, we can accomplish that the ERPSM is effortless, effective, and practical for solving numerous further fractional order PDEs.

\section{Data Availability}

No data were used to support this study. 


\section{Conflicts of Interest}

The authors declare that they have no conflicts of interest.

\section{References}

[1] S. Kumar, A. Ahmadian, R. Kumar et al., "An efficient numerical method for fractional SIR epidemic model of infectious disease by using Bernstein wavelets," Mathematics, vol. 8, no. 4, pp. 558-570, 2020.

[2] A. Khalouta and A. Kadem, "A comparative study of Shehu variational iteration method and Shehu decomposition method for solving nonlinear Caputo time-fractional wavelike equations with variable coefficients," Applications and Applied Mathematics: International Journal, vol. 15, no. 1, pp. 24-41, 2020.

[3] K. M. Saad, M. M. Khader, J. F. Gómez-Aguilar, and D. Baleanu, "Numerical solutions of the fractional Fisher's type equations with Atangana-Baleanu fractional derivative by using spectral collocation methods," Chaos: An Interdisciplinary Journal of Nonlinear Science, vol. 29, no. 2, pp. 101-122, 2019.

[4] P. T. Toan, T. N. Vo, and M. Razzaghi, "Taylor wavelet method for fractional delay differential equations," Engineering with Computers, vol. 37, no. 1, pp. 231-240, 2021.

[5] A. Saadatmandi and M. Dehghan, "A new operational matrix for solving fractional-order differential equations," Computers \& Mathematics with Applications, vol. 59, no. 3, pp. 13261336, 2010.

[6] A. Khalouta and A. Kadem, "Fractional natural decomposition method for solving a certain class of nonlinear timefractional wave-like equations with variable coefficients," Acta Universitatis Sapientiae, Mathematica, vol. 11, no. 1, pp. 99116, 2019.

[7] V. Gupta and S. Gupta, "Application of homotopy analysis method for solving nonlinear Cauchy problem," Surveys in Mathematics and its Applications, vol. 7, no. 1, pp. 105-116, 2012.

[8] R. I. Nuruddeen and A. M. Nass, "Aboodh decomposition method and its application in solving linear and nonlinear heat equations," European Journal of Advances in Engineering and Technology, vol. 3, no. 7, pp. 34-37, 2016.

[9] H. Eltayeb and E. Abdeldaim, "Sumudu decomposition method for solving fractional delay dierential equations," Research in Applied Mathematics, vol. 1, no. 1, pp. 1-13, 2017.

[10] J. Ul Rahman, D. Lu, M. Suleman, J. H. He, and M. Ramzan, "He-Elzaki method for spatial diffusion of biological population," Fractals, vol. 27, no. 5, pp. 112-121, 2019.

[11] S. Momani, O. Abu Arqub, M. m. Abu Hammad, and Z. S. Abo-Hammour, "A residual power series technique for solving systems of initial value problems," Applied Mathematics \& Information Sciences, vol. 10, no. 2, pp. 765-775, 2016.

[12] M. Delkhosh and K. Parand, "Generalized pseudospectral method: theory and applications," Journal of Computational Science, vol. 34, pp. 11-32, 2019.

[13] K. Parand and M. Delkhosh, "An effective numerical method for solving the nonlinear singular Lane-Emden type equations of various orders," Jurnal Teknologi, vol. 79, no. 1, 2017.

[14] R. Shah, H. Khan, S. Mustafa, P. Kumam, and M. Arif, "Analytical solutions of fractional-order diffusion equations by natural transform decomposition method," Entropy, vol. 21 , no. 6, pp. 557-570, 2019.
[15] H. Ahmad, T. A. Khan, I. Ahmad, P. S. Stanimirović, and Y. M. Chu, "A new analyzing method for nonlinear time fractional Cauchy reaction-diffusion model equations," Results in Physics, vol. 19, no. 8, pp. 462-474, 2020.

[16] N. F. Britton, Reaction-Diffusion Equations and Their Applications to Biology, Academic Press, New York, NY, USA, 1998.

[17] R. S. Cantrell and C. Cosner, Spatial Ecology via Reaction-Diffusion Equations. Wiley Series in Mathematical and Computational Biology, Wiley, New York, NY, USA, 2003.

[18] P. Grindrod, The Theory and Applications of Reaction-Diffusion Equations, Oxford University Press, Oxford, UK, 2nd edition, 1996.

[19] H. Gul, H. Alrabaiah, S. Ali, K. Shah, and S. Muhammad, "Computation of solution to fractional order partial reaction diffusion equations," Journal of Advanced Research, vol. 25, no. 19 , pp. $31-38,2020$.

[20] M. S. H. Chowdhury and I. Hashim, "Analytical solution for Cauchy reaction-diffusion problems by homotopy perturbation method," Sains Malaysiana, vol. 39, no. 3, pp. 495-504, 2010.

[21] S. Ali, S. Bushnaq, K. Shah, and M. Arif, "Numerical treatment of fractional order Cauchy reaction diffusion equations," Chaos, Solitons \& Fractals, vol. 103, no. 12, pp. 578-587, 2017.

[22] K. Wang and S. Liu, "A new Sumudu transform iterative method for time-fractional Cauchy reaction-diffusion equation," Springer Plus, vol. 5, no. 1, pp. 1-20, 2016.

[23] S. Kumar, A. Kumar, S. Abbas, M. Al Qurashi, and D. Baleanu, "A modified analytical approach with existence and uniqueness for fractional Cauchy reaction-diffusion equations," Advances in Difference Equations, vol. 2020, no. 1, 13 pages, 2020.

[24] K. Hosseini, M. Ilie, M. Mirzazadeh, and D. Baleanu, "An analytic study on the approximate solution of a nonlinear time-fractional Cauchy reaction-diffusion equation with the Mittag-Leffler law," Mathematical Methods in the Applied Sciences, vol. 44, no. 8, pp. 6247-6258, 2021.

[25] S. A. Lima, M. Kamrujjaman, and M. S. Islam, "Numerical solution of convection-diffusion-reaction equations by a finite element method with error correlation," AIP Advances, vol. 11, no. 8, pp. 85-98, 2021.

[26] T. M. Elzaki, "The new integral transform Elzaki transform," Global Journal of Pure and Applied Mathematics, vol. 7, no. 1, pp. 57-64, 2011. 